\title{
In-vivo Endoscopic Visualization of Pain Generators in the Lumbar Spine Anthony $T$ Yeung ${ }^{1,2^{*}}$
}

${ }^{1}$ Desert Institute for Spine Care Phoenix, Arizona, USA

${ }^{2}$ Department of Endoscopic Surgery, University of New Mexico School of Medicine, Albuquerque, New Mexico, USA

*Corresponding author: Anthony T Yeung, Desert Institute for Spine Care, Phoenix, Arizona, USA, Tel: +1 602-944-2900; E-mail: ayeung@sciatica.com Rec Date: August 09, 2017; Acc Date: August 22, 2017; Pub Date: August 26, 2017

Copyright: (C) 2014 Yeung AT. This is an open-access article distributed under the terms of the Creative Commons Attribution License, which permits unrestricted use, distribution, and reproduction in any medium, provided the original author and source are credited.

\section{Abstract}

Introduction: Traditional interventional pain management only provides temporary relief that depend on the patient's natural healing to mitigate pain. Visualizing the patho-anatomy with an endoscope targeting the pathoanatomy by interventional needle trajectories, however, has opened the door for surgical decompression and ablation of the pain generators. Endoscopic spine surgery is effective using mobile cannulas to target the pain source facilitated by surgical visualization and decompression and ablation using an endoscope. New instrumentation, techniques, specially configured endoscopes, access cannulas, RF and laser modalities all facilitate effective surgical treatment of the pain generator. While traditional translaminar surgical approaches provide open access to spinal pathology, there are conditions better suited for an endoscopic approach, especially when the surgeon can add intradiscal therapy using the transforaminal or translaminar approach. When a surgeon combines interventional techniques with endoscopic visualization, additional effective steps in the treatment algorithm are available. The purpose of this paper is to demonstrate that the physiology of pain can be visualized, and treated surgically as the path-anatomy of a pain generator.

Materials and method: In endoscopic transforaminal surgery, the Yeung Endoscopic Spine Surgery ${ }^{\mathrm{TM}}$ (YESS ${ }^{\mathrm{TM}}$ ) technique, is utilized: 1 . Needle and cannula placement for optimal instrument placement is calculated from skin marking drawn on the skin from the PA and Lateral C-arm image. A similar needle trajectory is utilized for diagnostic and therapeutic injections as a diagnostic precursor that helps predict the success of transforaminal endoscopic surgical intervention. 2. Injection of non-ionic radio-opaque contrast will create a foraminal epidural gram and produce epidural patterns that outline foraminal patho-anatomy such as HNP; central and lateral recess stenosis, and other pathologies from the epiduralgram pattern. 3. Evocative chromo-discography ${ }^{\mathrm{TM}}$ is performed to provide a normal or abnormal discogram pattern that helps correlate the patho-anatomy of discogenic pain. Disc and foraminal decompression is aided by vital tissue staining. 5. Endoscopic foraminoplasty decompresses the lateral recess and visualizes the exiting and traversing nerve in the axilla containing the Dorsal Root Ganglion (DRG), In addition, other anomalous path-anatomy not suspected or identified by traditional imaging can be visualized with the endoscope. 6 . Surgical exploration of the epidural space. 7. Probe the "hidden zone" of Mac Nab under local anesthesia with a capability for the patient to provide back to the surgeon during surgery while mildly sedated or without sedation under local anesthesia. 8. Using a biportal or multiple portal techniques for out-side in or inside-out removal of extruded and sequestered nucleus pulposus and other patho-anatomy. 9. Dorsal and foraminal visualized rhizotomy of the branches of the dorsal ramus to denervate the facet joint. A database of over 10,000 surgical cases utilizing jpeg and MP4 video imaging illustrate the painful conditions most suitable and also possible with endoscopic surgery.

Results: The transforaminal endoscopic technique will allow surgical access to the lumbar spine for treatment of a wide spectrum of painful degenerative conditions. There are, moreover, conditions where the endoscopic foraminal approach has advantages over traditional surgical approaches. These conditions are: 1. Discitis 2. Far lateral foraminal and extraforaminal HNP, especially at L5-S1, 3. Upper lumbar HNP 4. Lateral foraminal stenosis. 5. Discogenic pain from toxic annular tears 6 . Visualizing the pain generators responsible for failed back surgery syndrome (FBSS). 7. When anomalous nerves such as furcal nerves are visualized, judgment must be used to determine whether the nerves can be avoided or ablated. Avoiding the nerves my cause failed back surgery syndrome by failing to remove the source of pain in the "hidden zone", or ablation can resolve the cause of pain from these branches of spinal nerves, also described as conjoined nerves. If the nerve does not hurt on probing or thermal stimulation, it is usually safe to ablate the nerve, with the risk of temporary dysesthesia requiring time to resolve, or the use of transforaminal steroid blocks and sympathetic blocks. Repeat surgical attempts to further decompress the foramen is discouraged as the symptoms and any effect of weakness may worsen or become permanent.

Conclusion: New surgical skills are needed for spine surgeons to incorporate endoscopic spine surgery in their practice. Incorporating interventional pain management techniques as a surgical as well, and not just as a diagnostic procedure confirmed by the results of rational treatment of the patho-anatomy under local anesthesia helps marry 


\begin{abstract}
the basic science of surgical micro-anatomy with surgical results. This provides additional clinical information that facilitates surgical intervention. New surgical procedures focusing on intradiscal therapy, disc augmentation, biologics, annular modulation, and tissue neuromodulation are all well suited for the minimally invasive approach. Endoscopic foraminal access to the lumbar spine will open the door to for true minimally invasive access to the lumbar spine without affecting and destabilizing the dorsal muscle column. Formal training or mentorship is needed to make this technology mainstream. New evolving technology facilitated by robotics and biologics will help evolve this procedure in the near future.
\end{abstract}

Keywords: Endoscopic spine surgery; Selective Endoscopic Discectomy ${ }^{\mathrm{TM}}$; Evocative Chromodiscography ${ }^{\mathrm{TM}}$; Thermal annuloplasty; Toxic annular tears; Anomalous furcal and sympathetic nerves; Pedunculated synovial cysts; Foraminal osteophytes

\section{Introduction}

There is a crisis of affordability in spine care delivery that may be mitigated by the ability to treat pain generators in the lumbar spine by transforaminal visualization and decompression of the pain generators with an endoscope $[1,2]$.

Interventional pain management, often the first line of invasive treatment provides temporary relief that depend on natural healing to mitigate pain. Visualizing the patho-anatomy with an endoscope targeting the patho-anatomy by interventional needle trajectories, however, has opened the door for surgical decompression and ablation of the pain generators $[3,4]$. Endoscopic spine surgery is effective using mobile cannulas to target the pain source as a visualized surgical pain management procedure. New instrumentation and techniques, specially configured cannulas and endoscopes, facilitate effective surgical treatment of the pain generator. While traditional translaminar surgical approaches provide open access to spinal pathology, there are conditions better suited for a transforaminal endoscopic approach, especially when the surgeon can add intradiscal therapy $[5,6]$. When a surgeon combines interventional techniques with endoscopic visualization, additional effective steps in the treatment algorithm are available. The purpose of this paper is to demonstrate that the physiology of pain can be visualized as a pain generator.

\section{Materials and Methods}

Endoscopic foraminal surgery (The YESS ${ }^{\mathrm{TM}}$ ) technique, is utilized: 1. Needle and cannula placement for optimal instrument placement is calculated from coordinate lines drawn on the skin from the C-Arm image. The needle trajectory is utilized for diagnostic and therapeutic injections as a precursor to endoscopic surgical intervention. 2. Injection of non-ionic radio-opaque contrast will create a foraminal epidural gram and produce epidural patterns that outline foraminal patho-anatomy such as HNP, central and lateral recess stenosis. 3 . Evocative chromo-discography is performed to confirm discogenic pain [7-9]. Disc and foraminal decompression is aided by vital tissue staining. 5. Endoscopic foraminoplasty decompresses the lateral recess and visualizes the exiting and traversing nerve containing the dorsal root ganglion (DRG) in the axilla including other patho-anatomy. 6 . Diagnostic and surgical exploration of the epidural space. 7. Probe the hidden zone of Mac Nab under local anesthesia. 8. Using the biportal technique for inside out and outside in removal of extruded and sequestered nucleus pulposus. 9. Dorsal visualized rhizotomy of the branches of the dorsal ramus to denervate the facet joint [10-13]. A database of over 10,000 surgical cases utilizing jpeg and MP4 video imaging illustrate the painful conditions most suitable for foraminal endoscopic surgery. This is an endoscopically visualized surgical procedure that requires specialized surgical training and not just a fluoroscopically guided pain management procedure. Many different endoscope systems with other leading key opinion leaders are available.

\section{Results}

The transforaminal endoscopic and translaminar technique will allow surgical access to the lumbar spine for treatment of a wide spectrum of painful degenerative conditions.

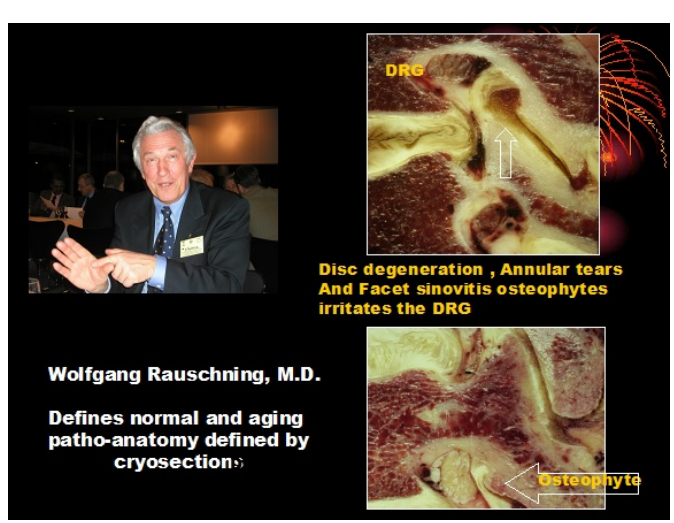

Figure 1a: Patho-anatomy in the lumbar spine as described by Wolfgang Rauschning in cadaver cryosections. The dorsal root ganglion is implicated by Dr Rauschning in his publications and lectures.

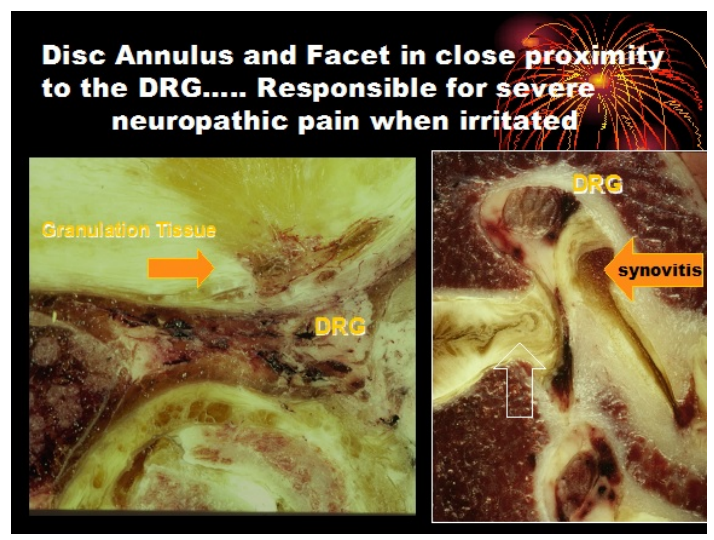

Figure 1b: Close-up view of the patho-anatomy in the annulus, facet, the facet joint causing synovitis, synovial cysts, and osteophyte impingement on the DRG. 
Citation: Yeung AT (2017) In-vivo Endoscopic Visualization of Pain Generators in the Lumbar Spine. J Spine 6: 385 . doi: $10.4172 / 2165-7939.1000385$

Page 3 of 5

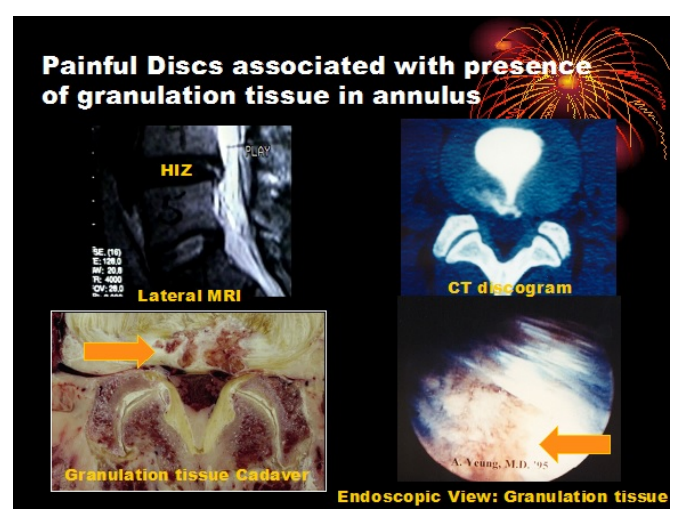

Figure 2a: Correlation of MRI imaging with granulation tissue found in symptomatic annular tears.

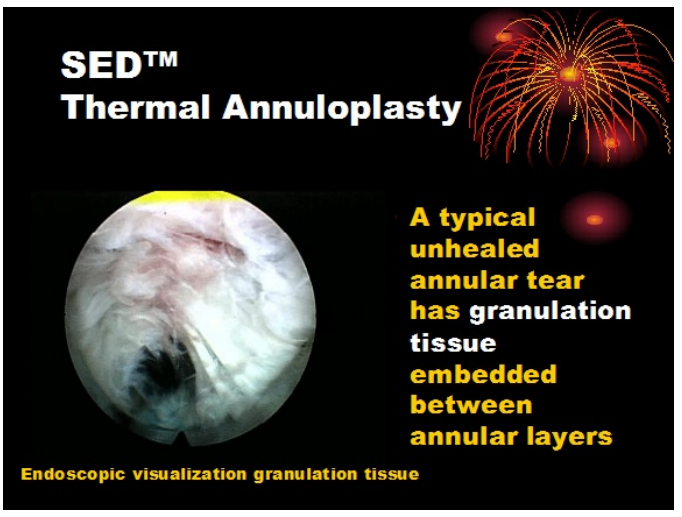

Figure 2b: Endoscopic intradiscal view of painful granulation tissue of pain sensitive granulation tissue in the annular tear.

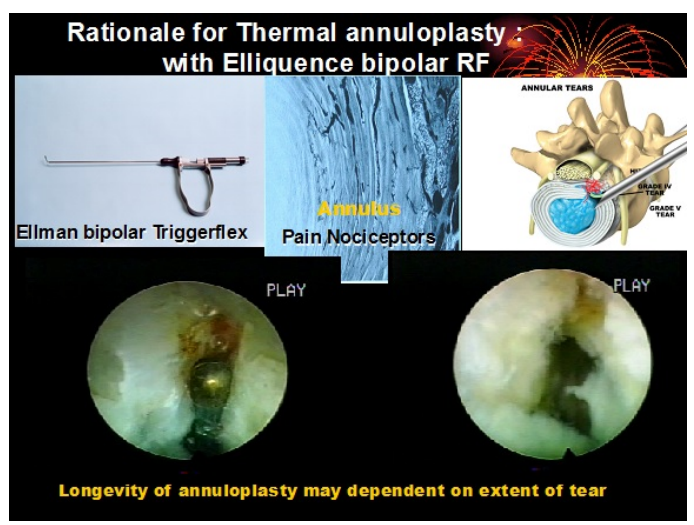

Figure 3: Illustration of the thermal annuloplasty concept in the trademarked selective endoscopic discectomy囚procedure by Yeung, on ablation of the endoscopically visualized annular tear with uniportal and biportal techniques.

The most common conditions responsible for "common low back pain" and sciatica from "Toxic Annular tears" (Figures 1 and 2).
These tears emanating from annular tears usually involve sensitization of the dorsal root ganglion, the (DRG) (Figure 3).

Ablation of postganglionic nerves by the DRG may cause symptoms of sympathetic dystrophy and cannot always be avoided (Figures $4 \mathrm{a}$ and $4 b)$.
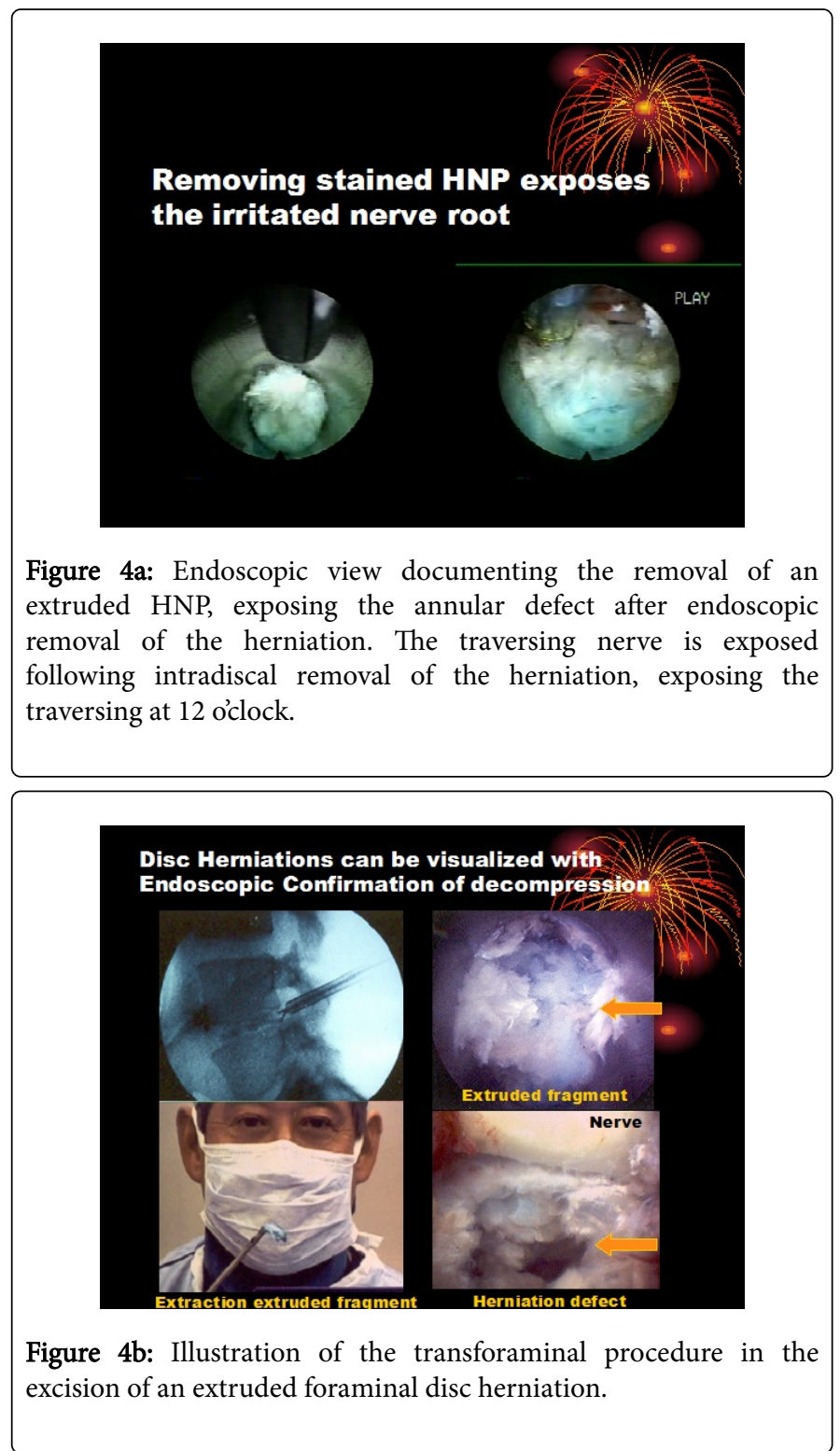

This is a known risk of irritation of the dorsal root ganglion by any aging condition involving the DRG, including surgical irritation. This is an unavoidable risk [14,15] (Figures 5 and 6). 
Citation: Yeung AT (2017) In-vivo Endoscopic Visualization of Pain Generators in the Lumbar Spine. J Spine 6: 385. doi: $10.4172 / 2165-7939.1000385$

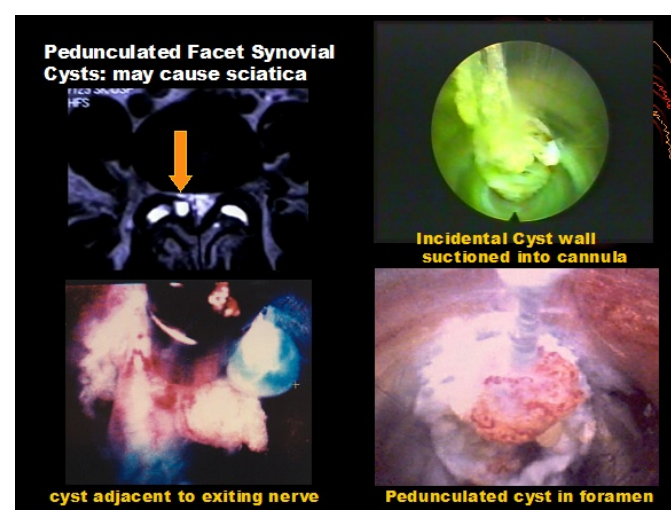

Figure 5: Pedunculated synovial cysts are sometime visualized endoscopically. Cysts may be suspected on MRI or is visualized incidentally, but contribute to sciatica.

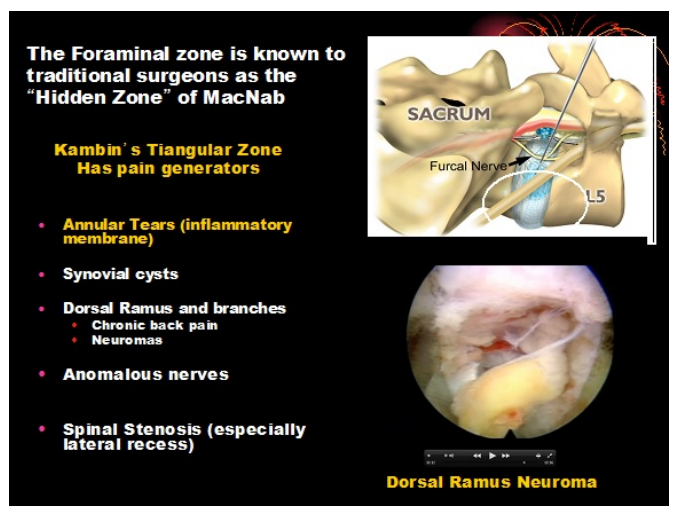

Figure 6: The hidden zone in the foramen, especially in the axilla between the traversing and exiting nerve contain patho-anatomic pain generators that are rarely seen by traditional surgeons who decompress with the translaminar approach.

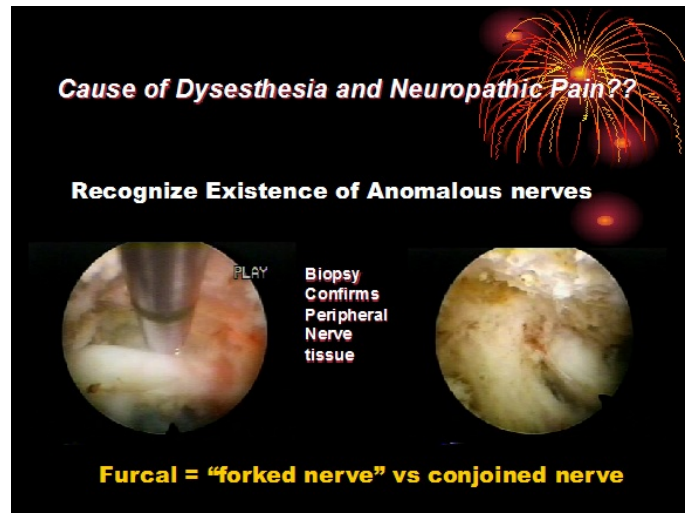

Figure 7: Anomalous nerves and variations of normal anatomy is often visualized in the foramen with the endoscope.

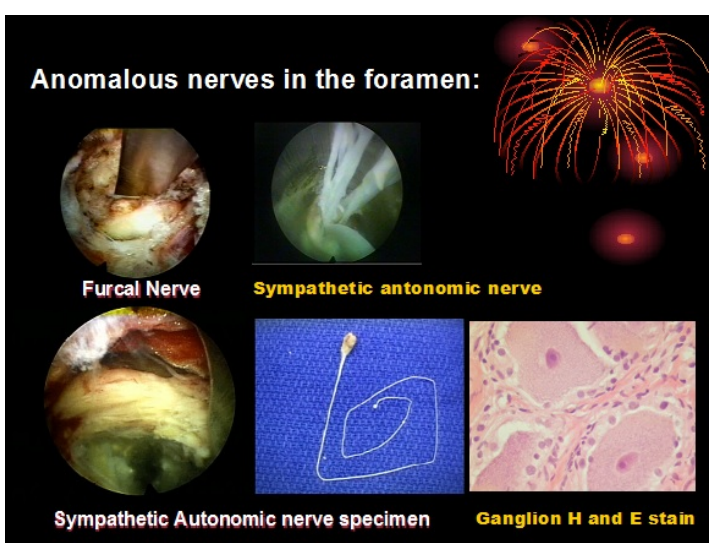

Figure 8: This anomalous nerve can be a branch of the spinal nerves or anomalous sympathetic nerves encountered during endoscopic foraminal decompression. When these pain-producing nerves are biopsied, they can look like spinal nerves or sympathetic nerves that contain ganglions are seen in autonomic and sympathetic nerves. This may explain the source of dysesthesia and sympathetic dystrophy. It is not always possible to identify a branch of the spinal nerve, called furcal nerves, with branches of the dorsal ramus that is a pure sensory nerve. If stimulation does not cause pain, and if the nerve branch is small, any post-op pain or weakness is almost always temporary unless there are systemic or individual patient comorbidities. When dysesthesia occurs either as an immediate or delayed response to ablation, the dysesthesia may take up to one year to resolve, as it is near the Dorsal root ganglion. This evokes a sometimes severe response similar to the complex regional syndrome of sympathetic dystrophy. The dysesthesia is best treated early with transforaminal epidural blocks and sympathetic block, taking an average to three blocks in succession to get symptom resolution.

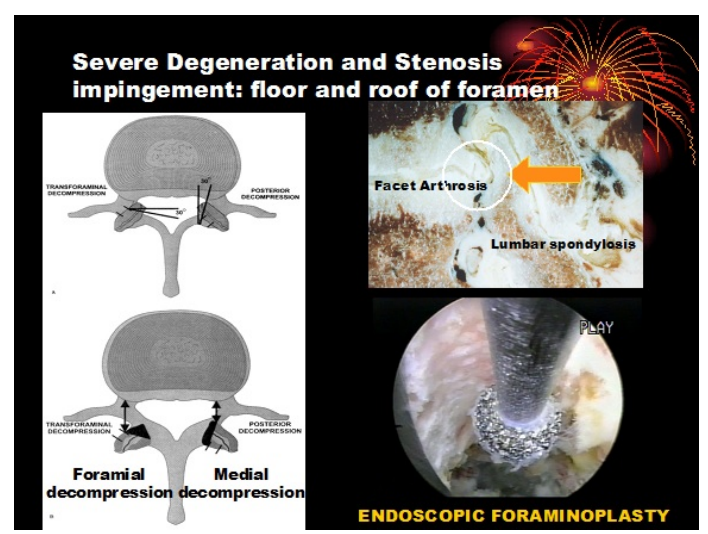

Figure 9a: Foraminal stenosis can be visualized as the absence of fat in the foramen or in direct visualization of foraminal osteophytes impinging on the exiting nerve in the foramen. Endoscopic foraminoplasty will relieve pain from osteophyte impingement.

There are, moreover, conditions where the endoscopic foraminal approach has advantages over traditional surgical approaches. These 
conditions are: 1 . Discitis 2. Far lateral foraminal and extraforaminal HNP, especially at L5-S1, 3. Upper lumbar HNP 4. Lateral foraminal stenosis (Figures 7 and 8 ).

5. Visualizing the pain generators responsible for FBSS. Conditions 1-4 can be treated with traditional translaminar decompression, but FBSS has pain generators that is routinely missed by traditional surgeon because the causes are in the "hidden zone" of MacNab between the traversing and exiting nerve [16] (Figures 9a and 9b).

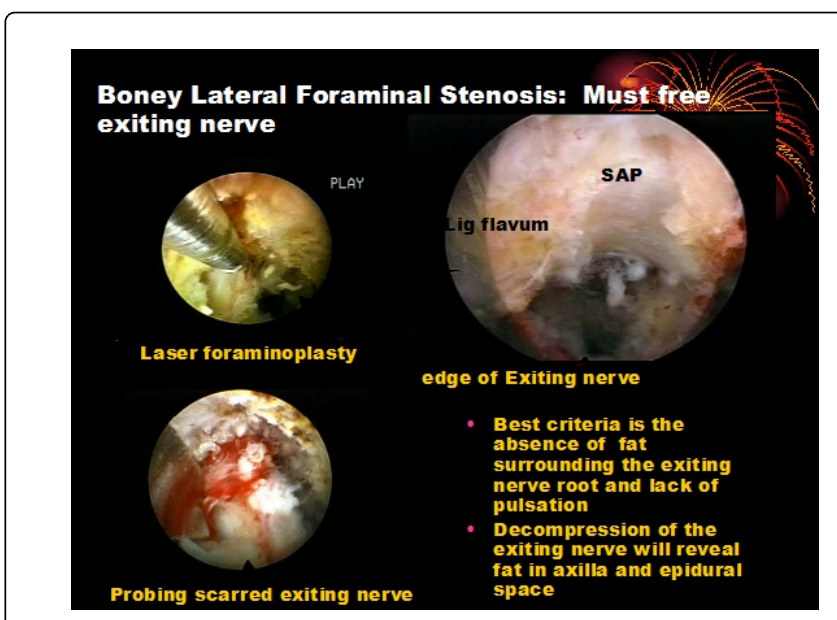

Figure 9b: Endoscopic view of foraminal stenosis.

\section{Discussion}

There are basically three techniques with two modern pioneer opinion leaders, Anthony Yeung promoting the "inside out" philosophy and technique, the YESS and Vertebris System, and Thomas Hoogland the developer of the Thessys system. followed by the Maxmore system. The YESS and Vertebris system, with a philosophy and technique emphasizing visualizing intradiscal patho-anatomy and visualization of the pain generators is discussed here.

\section{Conclusion}

New surgical skills are needed for spine surgeons to incorporate endoscopic spine surgery in their practice. Incorporating interventional pain management needle techniques as a surgical as well as just a diagnostic procedure is confirmed by treatment of the visualized patho-anatomy under local anesthesia as a new form of evidence based medicine. The Yess technique helps marry the basic science of microanatomy brings additional clinical information that facilitates surgical intervention. New surgical procedures focusing on intradiscal therapy, disc augmentation, biologics, annular modulation, and neuromodulation are all well suited for this minimally invasive approach. Endoscopic foraminal access to the lumbar spine will open the door to for true minimally invasive access to the lumbar spine without affecting and destabilizing the dorsal muscle column. Formal training in the academic or private setting, and mentorship is necessary to make this technology mainstream.

\section{References}

1. Yeung AT, Gore SR (2001) Evolving methodology in treating discogenic back pain by Selective Endoscopic Discectomy (SED) and thermal annuloplasty. J Minim Invasive Spine Surg Tech 1: 8-16.

2. Gore SR, Yeung AT (2003) Identifying sources of discogenic pain. J Minim Invasive Spine Surg Tech 3: 21-24.

3. Yeung AT, Tsou PM (2002) Posterolateral endoscopic excision for lumbar disc herniation: Surgical technique, outcome, and complications in 307 consecutive cases. Spine (Phila Pa 1976) 27: 722-731.

4. Yeung AT (2000) Minimally invasive surgery with the Yeung endoscopic spine system (YESS). Surg Tech Int 8: 267-27730.

5. Yeung AT, Yeung CA (2003) Advances in endoscopic disc and spine surgery: The foraminal approach. Surgical Tech Int 11:253-261.

6. Yeung AT (2003) Percutaneous endoscopic discectomy: The Posterolateral Approach, Minimal Access Spine Surgery, (2nd edn), Lieberman R (ed), Quality Medical Publishing, Missouri, USA.

7. Yeung AT, Gore SR (2011) In-vivo endoscopic visualization of pathoanatomy in symptomatic degenerative conditions of the lumbar spine II: Intradiscal, foraminal, and central canal decompression. Surg Technol Int 21: 299-319.

8. Yeung AT, Yeung CA (2006) In-vivo endoscopic visualization of pathoanatomy in painful degenerative conditions of the lumbar spine. Surg Technol Int 15: 243-256.

9. Yeung AT (2007) The evolution and advancement of endoscopic foraminal surgery: One surgeon's experience incorporating adjunctive technologies. SAS J 1: 108-117.

10. Yeung AT (2017) Transforaminal endoscopic decompression for painful degenerative conditions of the lumbar spine: A review of one surgeon's experience with Over 10,000 cases since 1991. J Spine Neurosurg 6: 2.

11. Anthony TY, Christopher AY (2017) Selective Endoscopic Lumbar Discectomy $\left(\mathrm{SED}^{\mathrm{m}}\right)$ and Thermal Annuloplasty for Discogenic back pain, disc herniations and sciatica in high performance athletes and physically active patients. Sports Injr Med 2017: 110.

12. Yeung AT (2017) Robotics in the MIS spine surgery arena: A new Role to Advance the adoption of endoscopic surgery as the least invasive spine surgery procedure. J Spine 6: 374.

13. Yeung A, Yeung CA (2017) Endoscopic identification and treating the pain generators in the lumbar spine that escape detection by traditional imaging studies. J Spine 6: 369.

14. Yeung A (2017) Failed back surgery syndrome: Endoscopic documentation of common causes by visualization of painful pathoanatomy in the hidden zone of the axilla containing the dorsal root ganglion and salvage treatment of neuropathic pain with DRG neuromodulation. SF J Neuro Sci 1: 1.

15. Yeung AT (2017) Delivery of spine care under health care reform in the United States. J Spine 6: 372.

16. Yeung AT (2017) A short note on minimally invasive lumbar spine surgery. J Spine 6: e127. 